Astronomy Letters, 2013, Vol. 39, No. 5, pp. 0-00 Printed 16 April 2013

\title{
Magnetically Active Stars in Taurus-Auriga: Evolutionary Status
}

\author{
K. N. Grankin \\ Crimean Astrophysical Observatory, Nauchny, Crimea, 98409 Ukraine \\ konstantin.grankin@rambler.ru
}

\begin{abstract}
We have analyzed a sample of 74 magnetically active stars toward the TaurusAuriga star-forming region. Based on accurate data on their basic physical parameters obtained from original photometric observations and published data on their proper motions, X-ray luminosities, and equivalent widths of the $\mathrm{H} \alpha$ and $\mathrm{Li}$ lines, we have refined the evolutionary status of these objects. We show that 50 objects are young stars with ages of 1-40 Myr and belong to the Taurus-Auriga star-forming region. Other 20 objects have a controversial evolutionary status and can belong to both Taurus-Auriga star-forming region and the Gould Belt. The remaining four objects with ages of 70-100 Myr belong to the zero-age main sequence. We have analyzed the relationship between the rotation period, mass, and age for 50 magnetically active stars. The change in the angular momentum of the sample stars within the first $40 \mathrm{Myr}$ of their evolution has been investigated. An active star-protoplanetary disk interaction is shown to occur on a time scale from 0.7 to 10 Myr.
\end{abstract}

Key words: stars - variable, properties, rotation, pre-main-sequence stars.

\section{INTRODUCTION}

The Taurus-Auriga star-forming region (SFR) includes a rich population of young premain-sequence (PMS) stars. According to Kenyon et al. (2008), the most complete sample of PMS stars in the Taurus-Auriga SFR contains 383 objects. This sample is represented by infrared objects or protostars, which are optically invisible because of strong absorption in the surrounding gas-dust clouds, T Tauri stars (TTS), and brown dwarfs. In turn, the subgroup of T Tauri stars includes both classical TTS (CTTS) with strong emission lines and significant infrared (IR) and ultraviolet (UV) excesses and weak-lined TTS (WTTS) with insignificant IR and UV excesses or without them. To all appearances, CTTS are at the stage of an active interaction with their accretion disks, while the disks around WTTS are either absent or not accretion ones. 
The listed objects form a rough age sequence with protostars as the youngest objects and WTTS as the oldest ones. The youngest protostars are concentrated in the densest parts of the molecular clouds; CTTS are located in both the clouds themselves and in the narrow dark bands that connect the largest clouds. WTTS show a lesser concentration to the clouds and can be slightly older than CTTS. Most of the PMS stars in the Taurus-Auriga SFR have ages of 1-3 Myr and only some of the SFR members have ages older than $3 \mathrm{Myr}$. If we take into account the fact that the molecular clouds in the SFR exist for about 10 Myr or more and assume that the star formation rate remained constant during the entire lifetime of the clouds, then we have to admit that the known PMS stars must represent only a small part of this association.

The absence of an observable population of older PMS stars with ages of about 3 Myr or older is characteristic not only for the Taurus-Auriga SFR but also for almost all of the neighboring SFRs (see, e.g., Carpenter 2000; Palla and Stahler 2000). Either star formation takes place during the short lifetime of the molecular clouds or the molecular clouds themselves exist only for several Myr. This problem has been discussed in the literature for almost three decades and is known as the problem of the absence of older PMS stars that have recently passed the TTS stage (post T Tauri stars or PTTS) (Herbig 1978; Feigelson 1996).

All of the stars later than the spectral type $\mathrm{F}$ and younger than 100 Myr manifest themselves as soft X-ray sources and they can be easily detected with the help of sensitive X-ray observatories. Several studies were undertaken to find new candidates for PMS stars among the multitude of X-ray sources toward the Taurus-Auriga SFR (see, e.g., Wichmann et al. 1996; Neuhäuser et al. 1997). These studies revealed a significant population of candidates for PMS stars that are widely dispersed over the Taurus-Auriga SFR and beyond. Since young stars exhibit small changes in their X-ray properties within the first $100 \mathrm{Myr}$ of their evolution, the X-ray data are insufficient to distinguish PMS stars with ages of 3-50 Myr from zero-age mainsequence (ZAMS) stars with ages of about $100 \mathrm{Myr}$. Thus, the sample of X-ray active stars can be heavily "contaminated" with field objects and additional spectroscopic observations are required to identify new PMS stars.

Using data from the ROSAT all-sky survey and spectroscopic data, Wichmann et al. (1996) investigated a region with a size of about 280 sq. degrees in the Taurus-Auriga SFR. As a result of this work, 76 candidates for PMS stars were identified (below referred to as Wichmann's list). Wichmann et al. (1996) used the following criteria to classify the stars as PMS: the presence of the Li I $\lambda 6707$ absorption line with an equivalent width $\geq 100 \mathrm{~m} \AA$ and the spectral type F or later. Most of these stars (72) were classified as WTTS based on the detection of weak emission or absorption in the $\mathrm{H} \alpha$ line $(\mathrm{EW}(\mathrm{H} \alpha) \leq 10 \AA)$. The remaining six PMS stars with strong $\mathrm{H} \alpha$ emission were classified as CTTS.

Martin and Magazzù (1999) disagreed with the classification criteria adopted by Wichmann et al. (1996). They pointed out that the detection of lithium in a late-type star is a necessary but not sufficient condition for it to be regarded as WTTS, because PTTS and low-mass members of young open clusters with ages of 30-200 Myr also show strong lithium lines (see Martin 1997). Martin and Magazzù (1999) used higher-resolution spectra for 35 stars from Wichmann's list to measure the equivalent widths of the lithium line and to revise the evolutionary status of these stars. They showed that their sample is a mixture of young (11 TTS and 8 PTTS) and older ZAMS stars. Wichmann et al. (2000) undertook another detailed study of 58 PMS candidates from Wichmann's list based on high-resolution echelle spectroscopy and proper motions. They found that approximately $60 \%$ of the stars from this sample could be regarded as PMS, while the remaining stars probably belong to the ZAMS. Wichmann et al. (2000) concluded that the PMS stars were probably associated with the Taurus-Auriga SFR, while the ZAMS stars could 
represent the population of older stars in the Gould Belt. Nevertheless, none of the objects from Wichmann et al. (2000) was included in the list of recognized members of the TaurusAuriga SFR published by Kenyon et al. (2008).

A long-term photometric monitoring of a representative sample of PMS stars had been performed as part of the ROTOR program at the Maidanak Astronomical Observatory in Uzbekistan for almost twenty years (1984-2006). As a result of the implementation of this program, more than $100000 U B V R$ measurements were obtained for 370 objects in various SFRs. The final results of our photometric observations for 72 CTTS and 48 WTTS are presented in Grankin et al. (2007) and Grankin et al. (2008), respectively. There were several tens of PMS stars from the Taurus-Auriga SFR among the observed objects: 34 CTTS and 40 WTTS. Since these stars are recognized members of the Taurus-Auriga SFR (see Kenyon et al. 2008), below we will call them "well-known PMS stars".

In addition, 60 candidates for PMS stars from Wichmann et al. (1996) were observed at the Maidanak Astronomical Observatory. More than 5000 BVR measurements were obtained for these stars during 1994-2006. Since the objects from Wichmann's list were not included in the list of recognized PMS stars from the Taurus-Auriga SFR published by Kenyon et al. (2008), below we will call these objects "candidates for PMS stars".

Previously (Grankin 2013a), we analyzed homogeneous long-term photometric observations of 28 well-known PMS stars published in Grankin et al. (2008) and 60 candidates for PMS stars from Wichmann's list toward the Taurus-Auriga SFR. We were able to determine reliable luminosities, radii, masses, and ages only for 74 of the 88 sample stars. In addition, 62 stars from this sample were shown to exhibit periodic light variations due to the phenomenon of spotted rotational modulation.

This paper is a logical continuation of our studies of a representative sample of stars begun in Grankin (2013a). Our main goals are: (1) to refine the evolutionary status of the candidates for PMS stars and to compare it with the evolutionary status of the well-known PMS stars from the Taurus-Auriga SFR; (2) to analyze the possible relationship between the rotation period, mass, and age for all sample stars; and (3) to investigate the change in the angular momentum of the sample stars within the first $40 \mathrm{Myr}$ of their evolution.

\section{PUBLISHED DATA}

To successfully solve the formulated problems, we collected published data on the proper motions, $\mathrm{H} \alpha$ and Li I (6707 $\AA$ ) line equivalent widths, and X-ray luminosities for all sample stars. The proper motions were taken from Ducourant et al. (2005) and are given in columns 2 and 3 of Table 1 . The data on the Li I (6707 $\AA$ ) and $\mathrm{H} \alpha$ line equivalent widths were compiled from the following papers: Herbig et al. (1986), Hartmann et al. (1987), Walter et al. (1988), Strom et al. (1989), Gomez et al. (1992), Magazzù et al. (1992), Martin et al. (1994), Martin and Magazzù (1999), Wichmann et al. (2000), Basri et al. (1991), and Nguyen et al. (2009). The median Li I (6707 $\AA$ ) and $\mathrm{H} \alpha$ line equivalent widths are given in columns 4 and 5 of Table 1. The X-ray luminosities were taken from Stelzer and Neuhäuser (2001) and Güdel et al. (2007) and are given in the last column. Table 2 presents analogous data for the objects from our previous paper (Grankin 2013a) that, for one reason or another, have no reliable data on their luminosities, radii, masses, or ages. 
Table 1: Published data for the sample stars

\begin{tabular}{|c|c|c|c|c|c|c|c|c|c|c|c|}
\hline W96 & $\begin{array}{l}\mu_{\alpha} \cos \delta \\
\operatorname{mas} / \mathrm{yr}\end{array}$ & $\begin{array}{c}\mu_{\delta} \\
\mathrm{mas} / \mathrm{yr}\end{array}$ & $\begin{array}{c}\mathrm{W}(\mathrm{Li}) \\
\AA\end{array}$ & $\begin{array}{c}\mathrm{W}(\mathrm{H} \alpha) \\
\AA\end{array}$ & $\begin{array}{c}\mathrm{Lx}, 10^{30} \\
\operatorname{erg~s}^{-1}\end{array}$ & W96/name & $\begin{array}{l}\mu_{\alpha} \cos \delta \\
\text { mas } / y r\end{array}$ & $\begin{array}{c}\mu_{\delta} \\
\operatorname{mas} / \mathrm{yr}\end{array}$ & $\begin{array}{c}\mathrm{W}(\mathrm{Li}) \\
\AA\end{array}$ & $\underset{\AA}{\mathrm{W}(\mathrm{H} \alpha)}$ & $\begin{array}{l}\text { Lx, } 10^{30} \\
\operatorname{erg~s}^{-1}\end{array}$ \\
\hline 01 & 2 & -11 & 0.26 & 0.38 & 2.94 & 60 & & & 0.10 & 1.50 & 0.37 \\
\hline 02 & 3 & -14 & 0.45 & -0.25 & 1.06 & 62 & 0 & -18 & 0.40 & -0.05 & 0.72 \\
\hline 03 & 16 & -20 & 0.22 & 0.70 & 2.31 & 63 & 9 & -20 & 0.58 & -0.50 & 1.11 \\
\hline 04 & 4 & -17 & 0.35 & 0.64 & 4.58 & 64 & -2 & -17 & 0.31 & -0.29 & 1.11 \\
\hline 05 & 6 & -15 & 0.25 & 2.77 & 3.46 & 66 & -1 & -18 & & 2.44 & 4.37 \\
\hline 06 & 7 & -15 & 0.21 & 3.28 & 3.14 & 67 & 0 & -17 & 0.25 & 0.60 & 0.97 \\
\hline 07 & 7 & -21 & 0.59 & -4.60 & 3.54 & 68 & 11 & -27 & & 0.10 & 0.48 \\
\hline 08 & 18 & -53 & 0.26 & -0.20 & 2.31 & 70 & 12 & -18 & 0.30 & -7.00 & 1.45 \\
\hline 10 & 20 & -32 & 0.41 & 1.00 & 3.21 & 71 & 0 & -18 & 0.20 & 2.71 & 0.74 \\
\hline 11 & 3 & -11 & 0.30 & -3.70 & 1.35 & 73 & 11 & -17 & 0.45 & 0.30 & 0.94 \\
\hline 12 & 30 & -43 & 0.26 & -1.80 & 0.74 & 74 & 2 & -18 & 0.43 & -0.10 & 3.49 \\
\hline 13 & -1 & -18 & 0.41 & -0.35 & 1.33 & 75 & 8 & -25 & 0.44 & 0.03 & \\
\hline 14 & 8 & -16 & 0.17 & 1.10 & 0.82 & 76 & 12 & -18 & 0.40 & -0.06 & 3.3 \\
\hline 15 & 16 & -40 & 0.16 & 2.60 & 0.40 & Anon 1 & & & 0.48 & -2.50 & 1.45 \\
\hline 18 & 1 & -14 & 0.27 & 1.88 & 2.46 & TAP 31 & 8 & -27 & & 1.01 & 9.12 \\
\hline 23 & -7 & -22 & 0.37 & -0.57 & 0.47 & LkCa 1 & 16 & -29 & 0.56 & -3.40 & 0.11 \\
\hline 27 & 8 & -16 & 0.36 & 0.75 & 2.93 & LkCa 4 & 8 & -24 & 0.61 & -4.05 & 0.91 \\
\hline 29 & 2 & -25 & 0.34 & 1.35 & 4.99 & $\mathrm{LkCa} 5$ & 7 & -33 & 0.55 & -3.90 & 0.34 \\
\hline 30 & 1 & -17 & 0.16 & 2.73 & & LkCa 7 & 5 & -31 & 0.59 & -3.70 & 0.83 \\
\hline 31 & 29 & -28 & 0.14 & 1.20 & 3.53 & LkCa 14 & 4 & -21 & 0.60 & -0.90 & 1.07 \\
\hline 32 & 3 & -14 & & 1.39 & 1.87 & LkCa 16 & 10 & -17 & 0.44 & -4.00 & 1.12 \\
\hline 36 & -5 & -12 & 0.24 & 0.66 & 0.20 & LkCa 19 & 3 & -19 & 0.47 & -0.50 & 5.50 \\
\hline 37 & 8 & -16 & 0.54 & -1.80 & 0.21 & $\mathrm{LkCa} 21$ & 12 & -30 & 0.75 & -5.50 & 1.82 \\
\hline 39 & -13 & -6 & $<0.05$ & 2.30 & 1.02 & TAP 4 & 25 & -41 & 0.35 & -0.10 & \\
\hline 40 & 13 & -23 & 0.49 & -2.50 & 3.88 & TAP 9 & 20 & -48 & 0.34 & -1.60 & 0.78 \\
\hline 41 & 0 & -11 & 0.36 & 0.51 & 0.62 & TAP 26 & 9 & -15 & 0.57 & -1.05 & 0.89 \\
\hline 44 & 7 & -3 & 0.35 & 0.24 & 0.44 & TAP 35 & 2 & -15 & 0.28 & 1.35 & 2.57 \\
\hline 45 & -8 & -16 & 0.25 & -1.30 & 0.70 & TAP 40 & 10 & -28 & 0.15 & -0.29 & 0.38 \\
\hline 46 & 7 & -11 & 0.38 & -0.50 & 0.29 & TAP 41 & 11 & -18 & 0.66 & -0.53 & 0.95 \\
\hline 47 & 14 & -19 & 0.42 & -0.07 & 1.24 & TAP 45 & 16 & -13 & 0.60 & -0.70 & 0.35 \\
\hline 48 & 17 & -46 & 0.50 & -0.39 & 2.72 & TAP 50 & -51 & -12 & & -0.60 & \\
\hline 53 & & & $<0.07$ & 0.00 & 0.95 & TAP 57 & 2 & -26 & 0.58 & -1.05 & 0.78 \\
\hline 54 & 7 & -23 & 0.19 & -2.10 & 2.49 & V819 Tau & 9 & -32 & 0.62 & -2.50 & 0.79 \\
\hline 56 & 41 & -15 & $<0.05$ & -0.90 & 0.71 & V826 Tau & 13 & -22 & & -3.30 & 1.35 \\
\hline 57 & -4 & -9 & 0.20 & 1.85 & 0.91 & V827 Tau & 8 & -15 & 0.57 & -2.95 & 1.95 \\
\hline 58 & 1 & -18 & 0.28 & 1.40 & 2.14 & V830 Tau & -8 & -28 & 0.68 & -2.00 & 1.91 \\
\hline 59 & 16 & -23 & 0.47 & -5.00 & 0.90 & VY Tau & 12 & -20 & 0.52 & -4.90 & 0.98 \\
\hline
\end{tabular}


Table 2: Published data for the sample stars without reliable information about their luminosities, radii, masses, and ages

\begin{tabular}{c|c|c|r|r|r||r|c|r|r|r|c}
\hline \hline $\mathrm{W} 96$ & $\begin{array}{c}\mu_{\alpha} \cos \delta \\
\mathrm{mas} / \mathrm{yr}\end{array}$ & $\begin{array}{c}\mu_{\delta} \\
\mathrm{mas} / \mathrm{yr}\end{array}$ & $\begin{array}{c}\mathrm{W}(\mathrm{Li}) \\
\AA\end{array}$ & $\begin{array}{c}\mathrm{W}(\mathrm{H} \alpha) \\
\AA\end{array}$ & $\begin{array}{c}\mathrm{Lx}, 10^{30} \\
\mathrm{erg} \mathrm{s}^{-1}\end{array}$ & $\mathrm{~W} 96 / \mathrm{name}$ & $\begin{array}{c}\mu_{\alpha} \cos \delta \\
\mathrm{mas} / \mathrm{yr}\end{array}$ & $\begin{array}{c}\mu_{\delta} \\
\text { mas/yr }\end{array}$ & $\begin{array}{c}\mathrm{W}(\mathrm{Li}) \\
\AA\end{array}$ & $\begin{array}{c}\mathrm{W}(\mathrm{H} \alpha) \\
\AA\end{array}$ & $\begin{array}{c}\mathrm{Lx}, 10^{30} \\
\mathrm{erg} \mathrm{s}^{-1}\end{array}$ \\
\hline 09 & 16 & -17 & $<0.07$ & -0.10 & 2.64 & 55 & 12 & -18 & 0.36 & 1.40 & 3.66 \\
19 & 1 & -9 & 0.17 & 2.10 & 0.53 & 61 & & & 0.19 & 2.80 & 0.37 \\
28 & 11 & -27 & 0.40 & 0.40 & & 65 & & & & 3.80 & 0.31 \\
38 & 3 & -7 & & 2.10 & 0.41 & 72 & -6 & -12 & $<0.11$ & 0.90 & 1.23 \\
49 & 4 & -5 & 0.14 & 2.55 & 0.53 & LkCa 3 & 6 & -21 & 0.56 & -2.70 & 0.68 \\
50 & -2 & -21 & 0.23 & 2.88 & 0.70 & V410 Tau & 6 & -31 & 0.54 & -2.25 & 2.40 \\
51 & 2 & -15 & & 1.80 & 0.22 & V836 Tau & 11 & -10 & 0.57 & -7.70 & 0.74 \\
52 & -2 & 7 & & 1.00 & 0.52 & TAP 49 & 0 & -7 & 0.27 & 0.90 & 0.05 \\
\hline
\end{tabular}

\section{PROPER MOTION}

Figure 1 presents 70 objects from our sample with available proper motions from Ducourant et al. (2005). The well-known PMS stars and PMS candidates are designated by the gray and black colors, respectively. The objects with reliable physical parameters given in Table 1 are marked by the circles; the objects without reliable parameters presented in Table 2 are designated by the squares. To analyze the proper motions of the objects from our sample, we invoked additional data on the proper motions of 123 recognized members of the Taurus-Auriga SFR from the same paper by Ducourant et al. (2005). These are designated by the crosses on the plot. Using these data, we calculated the mean and standard deviation for the proper motions of the recognized members of the Taurus-Auriga SFR:

$$
\begin{aligned}
\mu_{\alpha} \cos \delta & =5.83 \pm 5.91 \mathrm{mas} \mathrm{yr}^{-1} \\
\mu_{\delta} & =-19.52 \pm 7.58{\mathrm{mas} \mathrm{yr}^{-1}} .
\end{aligned}
$$

The large ellipse in Fig. 1 bounds the $3 \sigma$ region around this mean. It can be seen from the figure that most of the stars from our sample are within this ellipse, i.e., their proper motions correspond to those of the recognized SFR members. At the same time, there are several objects, both among the stars of our sample and among the recognized SFR members, whose proper motions differ significantly from the mean proper motion of the SFR. Seven objects should be pointed out among the recognized SFR members from Kenyon's list: HBC 355, HBC 358, HBC 360, HBC 361, HBC 362, HBC 375, and HBC 418. Our list contains 10 such objects: W39, W31, W56, W12, W08, W48, W15, TAP 4, TAP 9, and TAP 50; 5 of these 10 stars (W08, W48, W15, TAP 4, and TAP 9) have proper motions close to those of the Pleiades members (enclosed in the small gray circle). Similar conclusions about the kinematic properties of these five stars from our sample were reached by Frink et al. (1997). In particular, they point out that TAP 4, TAP 9, W08, W15, and W48 are kinematic members of the Pleiades.

A detailed kinematic study of the Taurus-Auriga SFR was undertaken by Bertout and Genova (2006). They also determined the kinematic parallaxes for 15 PMS candidates from Wichmann's list and found that 8 of these 15 candidates are SFR members (W05, W06, W27, W32, W37, W62, W71, W74) and 7 others are not (W01, W07, W14, W29, W54, W58, W67). However, all these 15 stars have proper motions very close to the mean proper motion of the SFR (the deviations from the mean are less than $1 \sigma$ ). Therefore, we believe that all 15 stars 
are possible members of the Taurus-Auriga SFR. It should be noted that the proper motions of 13 objects from our sample without reliable data on their luminosities, radii, masses, and ages agree well with the mean proper motion of the SFR within $3 \sigma$ (see Table 2). Only one star, W52, constitutes an exception.

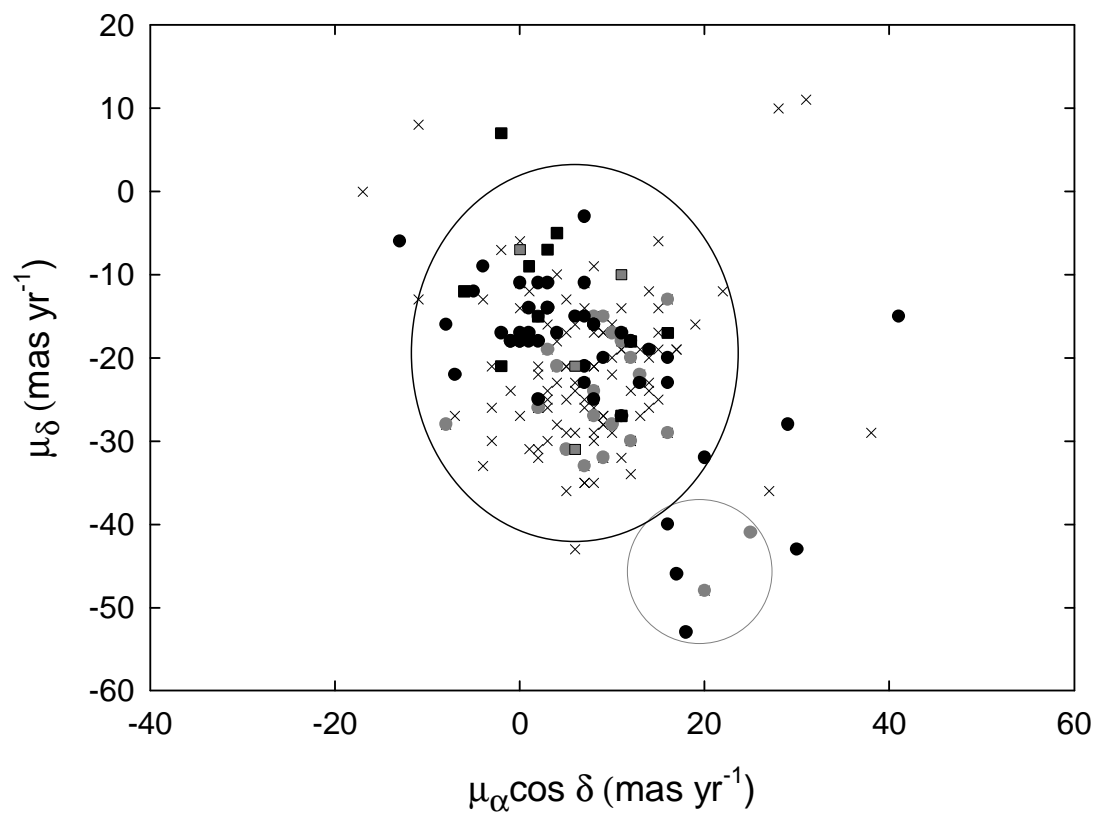

Figure 1: Proper motions for 23 well-known PMS and 48 PMS candidates from our sample (the gray and black circles, respectively). The recognized members of the Taurus-Auriga SFR from the list by Kenyon et al. (2008) are represented by the crosses. The large ellipse bounds the $3 \sigma$ region around the mean value of the proper motions for 123 recognized SFR members. Five stars with proper motions close to those of the Pleiades members are enclosed in the small gray circle. Fourteen stars without reliable data on their luminosities, radii, masses, and ages are designated by the gray (4 PMS) and black (10 PMS candidates) squares.

\section{LITHIUM LINE EQUIVALENT WIDTH}

In Fig. 2, the lithium equivalent width $(\mathrm{EW}(\mathrm{Li}))$ is plotted against the effective temperature $\left(T_{\text {eff }}\right)$ for the objects from our sample and the Pleiades stars. EW(Li) for the Pleiades stars were taken from Soderblom et al. (1993). The gray and black circles designate, respectively, 25 well-known PMS and 45 PMS candidates from our sample for which we were able to find EW(Li). The Pleiades stars are represented by the crosses. The dashed curve is the upper envelope for the sample of stars from the Pleiades. It is used as the boundary between the PMS and ZAMS stars (with an age of $\sim 100 \mathrm{Myr}$ ). If an object from our sample is located above the enveloping dashed line, then we deem it to be a fairly young object, with an age of less than 100 Myr.

It can be seen from the figure that 16 stars from our sample lie in the region of the diagram where the Pleiades stars are located. At the same time, a significant fraction of our objects (54 of 70) are in the region of young PMS stars. In addition to the main group of 70 stars, we marked 12 more objects without reliable data on their luminosities, radii, masses, and ages (see Table 2) for which EW(Li) are known. These are designated by the gray (4 PMS) and black (8 PMS candidates) squares. Seven stars from this group are located in the region of relatively old Pleiades stars (W9, W19, W49, W50,W61, W72, and TAP 49). 


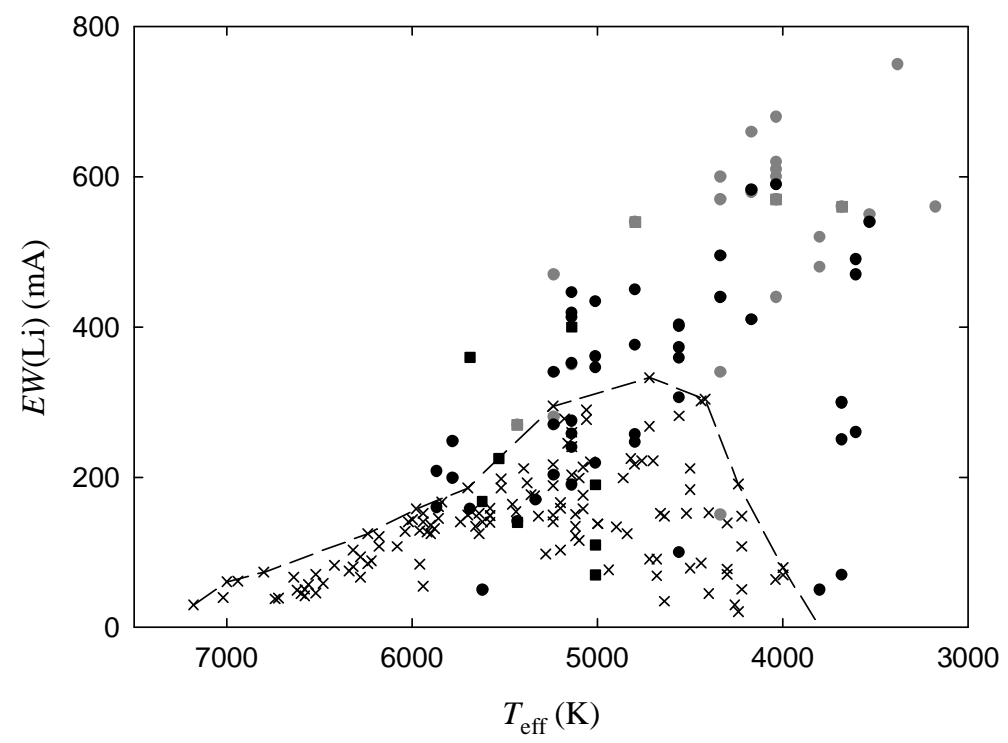

Figure 2: $\mathrm{EW}(\mathrm{Li})$ versus $T_{\text {eff }}$ for the Pleiades (crosses), 25 well-known PMS (gray circles), and 45 PMS candidates (black circles). Twelve objects without reliable data on their luminosities, radii, masses, and ages are designated by the gray (4 PMS) and black (8 PMS candidates) squares. The dashed curve indicates the upper envelope for the sample of stars from the Pleiades.

\section{AGE}

Previously (Grankin 2013a), we estimated the ages of the sample stars from the HertzsprungRussell (HR) diagram using evolutionary tracks from Siess et al. (2000). Figure 3 shows the age distribution (histogram) for the well-known PMS stars from our sample (Fig. 3a) and the PMS candidates (Fig. 3b). We excluded four objects with ages older than 70 Myr from consideration. The evolutionary status of these four objects is discussed in the next section.

It can be seen from the figure that the ages of the well-known PMS stars are, on average, younger than those of the PMS candidates. The age distribution for the well-known PMS is spiky, with its peak near 2 Myr. About half of the well-known PMS objects have an age of about 2 Myr; the remaining stars have ages in the range 4-18 Myr. The distribution for the PMS candidates is wider with three low peaks near 2, 14, and $22 \mathrm{Myr}$. In the next section, we refine the evolutionary status of the sample stars using their ages, lithium and $H \alpha$ line equivalent widths, and proper motions.

\section{EVOLUTIONARY STATUS}

As has been shown in the preceding section, there are young objects with ages of 1-10 Myr both in the group of well-known PMS stars and in the group of PMS candidates from Wichmann's list. There is good reason to suppose that such young objects are members of the Taurus-Auriga SFR. However, quite a few objects with ages of 10-20 Myr are present in both groups and there are even objects with ages of 20-45 Myr in the group of candidates for PMS stars. To classify the objects under study and to refine their evolutionary status, we invoked data not only on their ages but also on their proper motions and the lithium and $H \alpha$ line equivalent widths.

As a result of our analysis of the available data, we propose the following classification scheme. We classify all objects from our sample with ages up to $10 \mathrm{Myr}$ as WTTS candidates and those with ages of 11-50 Myr as PTTS candidates. An object has a very reliable evolu- 

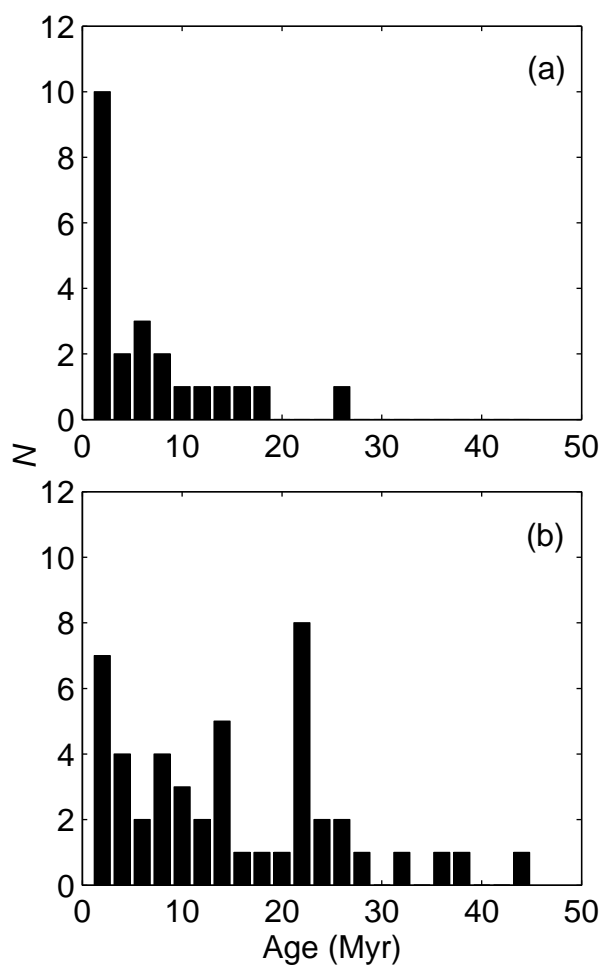

Figure 3: Age distribution for the well-known PMS stars (a) and PMS candidates from Wichmann's list (b).

tionary status, WTTS or PTTS, if: (1) it exhibits a fairly strong lithium line (lies above the region occupied by the Pleiades members in Fig. 2), (2) its $H \alpha$ line is in emission, and (3) its proper motion corresponds to that of the Taurus-Auriga SFR. We designate such an object as "wtts+" or "ptts+", depending on its age.

If one of the parameters does not meet the requirements described above or if there are no data for it, then we deem such an object to have a fairly reliable evolutionary status, WTTS or PTTS, and designate it as "wtts" or "ptts", depending on its age. If any two parameters do not meet the requirements described above or if there are no data for them, then we deem such an object to have an unreliable evolutionary status and designate it as "wtts?" or "ptts?". If none of the parameters, except the age, does not meet the requirements described above or if there are no data for all of these parameters, except the age, then we deem such an object to have a very unreliable evolutionary status and designate it as "wtts??" or "ptts??".

Before reaching conclusions about the evolutionary status of the candidates for PMS stars from our sample, we tested the proposed classification scheme on the group of well-known PMS stars from our sample. All 24 well-known PMS stars enter into the catalog by Herbig and Bell (1988) and were classified as young weak-lined objects (WTTS). According to the proposed classification scheme, 14 well-known PMS objects are very reliable WTTS ("wtts+"), two objects are marked as reliable WTTS ("wtts"), and two objects are possible WTTS ("wtts?"). Two objects were classified as very reliable PTTS ("ptts+"), two objects were marked as reliable PTTS ("ptts"), one object is a possible PTTS ("ptts?"), and one object (TAP 4) belongs to the Pleiades (for a discussion, see below).

Thus, 16 well-known PMS stars from our sample are reliable WTTS ("wtts+" and "wtts") and 4 stars are reliable PTTS ("ptts+" and "ptts"). If we turn to the most complete list of young stars in the Taurus- Auriga SFR published by Kenyon et al. (2008), then it can be noted that all 16 objects that we assign to reliable WTTS are present in this list, while the remaining 
four objects are absent. In other words, the classification scheme proposed here yields reliable results for the objects from our sample with a known evolutionary status, and we can apply this classification to the group of candidates for PMS stars from our sample whose evolutionary status has been actively discussed in recent papers (see the Introduction).

According to the proposed classification scheme, 28\% (14 of 50) of the PMS candidates are reliable WTTS ("wtts+" and "wtts") and 32\% (16 of 50) of the PMS candidates are reliable PTTS ("ptts+" and "ptts"). The remaining 34\% (17 of 50) have an unreliable evolutionary status. Thus, it can be asserted that $60 \%$ of the PMS candidates from Wichmann's list are reliable PMS stars (WTTS and PTTS) with ages of 1-40 Myr that can be members of the Taurus-Auriga SFR with a high probability. In general, our conclusions are consistent with those of Wichmann et al. (2000) with some exceptions. For example, among the 29 common stars designated by Wichmann et al. (2000) as PMS, we classified 28 objects as reliable WTTS and PTTS. Only one object (W15) has a contradictory classification. In contrast to Wichmann et al. (2000), we believe that it belongs to the ZAMS. Similar conclusions can also be reached about the objects that were classified as stars with a controversial evolutionary status. For example, among the 15 common stars designated by Wichmann et al. (2000) as ZAMS objects, we classified 12 stars as unreliable WTTS and PTTS. Only three objects (W07, W29, and W54) have a contradictory classification. In contrast to Wichmann et al. (2000), we classified these three stars as reliable WTTS. Our classification of these three stars is consistent with that of Martin and Magazzù (1999) and Bertout and Genova (2006).

The results of the proposed classification of the objects under study are presented in Table 3. Several objects do not fit into the proposed scheme because of their old ages. We are talking about W15, W39, W46, and TAP 4. The age of the first three objects is close to $100 \mathrm{Myr}$. Without any doubt, they belong to zero-age main-sequence (ZAMS) stars. We designated their status as "ZAMS". TAP 4 have a proper motion and age like those of the Pleiades stars. In addition, this star is close in space to the Pleiades and is characterized by a very rapid rotation $(\mathrm{P}=0.482$ day $)$. We designated its evolutionary status as "Pleiades".

To summarize, it can be said that there are 50 reliable PMS stars (30 WTTS and 20 PTTS) among the stars of our sample. The axial rotation periods are known for 35 objects from this group. At the same time, there are 20 stars with an unreliable evolutionary status, with the exception of three ZAMS objects and one object from the Pleiades. Wichmann et al. (2000) supposed that the objects with an unreliable evolutionary status could represent the population of older stars from the Gould Belt.

Here, we point out, however, that many properties of the 20 stars that have no reliable evolutionary status within our classification scheme are very similar to those of the 50 PMS stars with a reliable evolutionary status. All 20 stars with an unreliable evolutionary status are located toward the Taurus-Auriga SFR; their proper motions and age estimates correspond to those of the recognized SFR members. All these stars were discovered owing to their enhanced X-ray luminosity (see Tables 1 and 2) suggesting a solar-type activity. Indeed, 15 of the 20 stars with an unreliable evolutionary status exhibit the phenomenon of spotted rotational modulation, just as 35 stars from the group of reliable members of the Taurus-Auriga SFR. Using the known rotation periods and photometric data, we estimated the mean distance to these 15 stars (for the technique, see Grankin 2013a). It is $110 \pm 33 \mathrm{pc}$ and agrees satisfactorily with the mean distance to the Taurus-Auriga SFR $(\simeq 140 \mathrm{pc})$. All these circumstances allow the two subgroups of stars (with a reliable and unreliable evolutionary status) to be considered as one combined group of 70 young, magnetically active pre-main-sequence stars located toward the Taurus-Auriga SFR. In the next section, we analyze the possible relationship between the rotation period, mass, and age of these PMS objects. 
Table 3: Evolutionary status of the sample objects

\begin{tabular}{|c|c|c|c|}
\hline W96/name & Status & W96/name & Status \\
\hline 01 & wtts? & 60 & ptts?? \\
\hline 02 & ptts+ & 62 & ptts+ \\
\hline 03 & ptts? & 63 & wtts+ \\
\hline 04 & ptts & 64 & ptts \\
\hline 05 & ptts & 66 & ptts? \\
\hline 06 & ptts & 67 & ptts? \\
\hline 07 & wtts+ & 68 & ptts? \\
\hline 08 & ptts? & 70 & wtts+ \\
\hline 10 & ptts & 71 & ptts \\
\hline 11 & wtts+ & 73 & wtts \\
\hline 12 & wtts & 74 & ptts+ \\
\hline 13 & ptts+ & 75 & wtts \\
\hline 14 & ptts? & 76 & ptts+ \\
\hline 15 & ZAMS & Anon 1 & wtts \\
\hline 18 & ptts? & TAP 31 & wtts? \\
\hline 23 & ptts+ & $\mathrm{LkCa} 1$ & wtts+ \\
\hline 27 & ptts & $\mathrm{LkCa} 4$ & wtts+ \\
\hline 29 & wtts & $\mathrm{LkCa} 5$ & wtts+ \\
\hline 30 & ptts? & $\mathrm{LkCa} 7$ & wtts + \\
\hline 31 & ptts?? & $\mathrm{LkCa} 14$ & wtts+ \\
\hline 32 & ptts? & LkCa 16 & wtts + \\
\hline 36 & ptts? & LkCa 19 & ptts+ \\
\hline 37 & wtts+ & LkCa 21 & wtts+ \\
\hline 39 & ZAMS & TAP 4 & Pleiades \\
\hline 40 & wtts+ & TAP 9 & ptts \\
\hline 41 & ptts & TAP 26 & ptts+ \\
\hline 44 & ptts & TAP 35 & ptts? \\
\hline 45 & wtts+ & TAP 40 & ptts \\
\hline 46 & ZAMS & TAP 41 & wtts+ \\
\hline 47 & ptts+ & TAP 45 & wtts+ \\
\hline 48 & wtts & TAP 50 & wtts? \\
\hline 53 & wtts?? & TAP 57 & wtts+ \\
\hline 54 & wtts & V819 Tau & wtts+ \\
\hline 56 & wtts? & V826 Tau & wtts \\
\hline 57 & ptts? & V827 Tau & wtts+ \\
\hline 58 & ptts? & V830 Tau & wtts+ \\
\hline 59 & wtts+ & VY Tau & wtts+ \\
\hline
\end{tabular}




\section{ROTATION PERIOD, MASS, AND AGE}

Since the magnetically active stars in Taurus-Auriga have quite different masses, it is interesting to investigate the possible rotation period-mass relationship. We took the rotation periods, masses, and ages from our previous paper (Grankin 2013a). The sample of stars with known rotation periods was divided into four subgroups, depending on the mass. The condition that the number of objects be the same in all subgroups was met. Figure 4 presents the distributions (histograms) of rotation periods for each individual subgroup of stars. It can be seen from the figure that the stars with the lowest masses $\left(0.24<M<0.8 M_{\odot}\right)$ exhibit rotation periods in the entire range of detected periods. The stars from the second subgroup $\left(0.8<M<1.0 M_{\odot}\right)$ have predominantly short rotation periods, no more than 4 days. Only three stars from this subgroup exhibit periods in the range from 4 to 10 days. The third subgroup $\left(1.0<M<1.2 M_{\odot}\right)$ contains only one star with a period longer than 4 days. Finally, the relatively massive stars $\left(1.2<M<1.9 M_{\odot}\right)$ exhibit only short rotation periods, no more than 3 days. Thus, stars with short rotation periods are present in all four subgroups irrespective of the mass. In contrast, the number of stars with long rotation periods $\left(P_{\text {rot }}>4\right)$ decreases appreciably with increasing mass of the objects. For this reason, the more massive PMS stars from our sample rotate, on average, faster than the less massive ones. The mean rotation periods for the four subgroups as the mass increases are the following: 4.13, 3.06, 2.16, and 1.97 days, respectively.
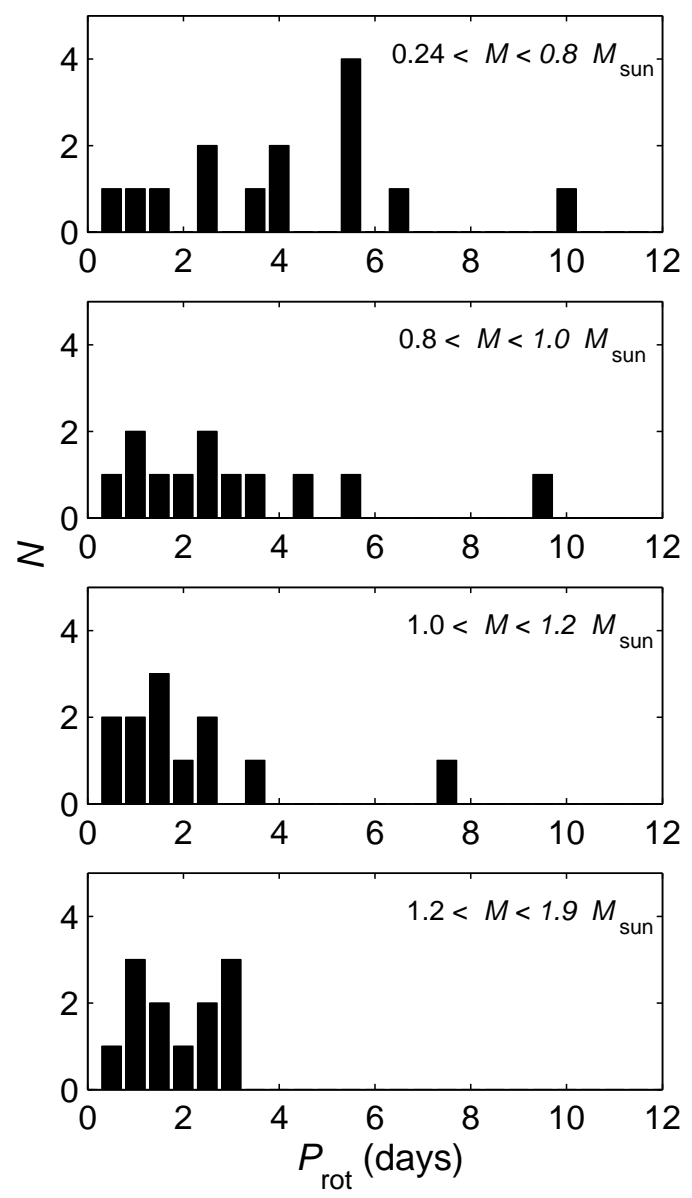

Figure 4: Distributions of rotation periods (histograms) for four subgroups of PMS stars with different masses. 
Figure 5 presents the distributions (histograms) of rotation periods for two different age subgroups of magnetically active stars: for 25 WTTS $(t<10 \mathrm{Myr})$ and 25 PTTS $(t>10 \mathrm{Myr})$. It can be seen from the figure that the younger PMS stars (WTTS) have both short $(P<5$ days) and long rotation periods in the range from 5 to 10 days. In contrast, the older PMS stars (PTTS) with ages of more than $10 \mathrm{Myr}$ exhibit only short rotation periods. In other words, the relatively old PMS stars with ages of more than 10 Myr rotate faster than the youngest stars with ages of less than $10 \mathrm{Myr}$. This result is in good agreement with the theoretical models that predict an increase in the rotation velocity (a decrease in the rotation period) as the PMS stars move toward the ZAMS. In the next section, we discuss in detail the evolution of angular momentum for the magnetically active stars from our sample.
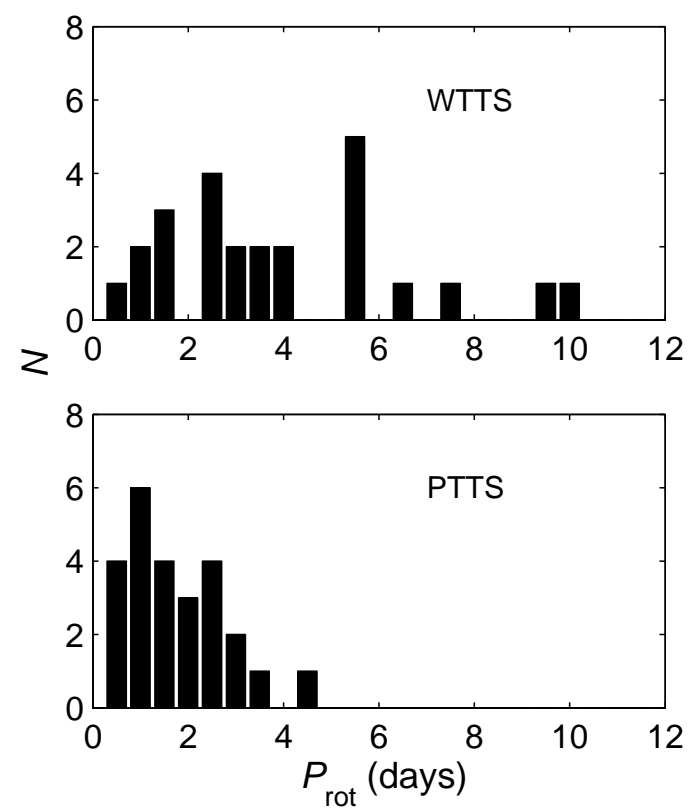

Figure 5: Distributions of rotation periods for $25 \operatorname{WTTS}(t<10 \mathrm{Myr})$ and 25 PTTS $(t>10 \mathrm{Myr})$.

\section{THE EVOLUTION OF ANGULAR MOMENTUM}

The spin-up of solar-type stars on PMS radiative tracks is believed to result from a decrease in the moment of inertia. As soon as the PMS stage ends, the moment of inertia subsequently remains essentially constant and a magnetized wind brakes the star during its MS evolution. Within the first 5-10 Myr of evolution, the star-protoplanetary disk interaction is believed to be the main cause of the angular momentum loss by PMS stars. The exact mechanism responsible for the angular momentum loss is still being actively discussed. At present, the two most probable mechanisms are considered: the removal of angular momentum through a stellar wind (see, e.g., Matt and Pudritz 2005) and an active interaction between the stellar magnetic field and the ionized gas in the inner protoplanetary disk regions (see, e.g., Konigl 1991; Collier Cameron and Campbel 1993).

It is more preferable to use the rotation periods than the equatorial rotation velocities to effectively test particular models for the evolution of angular momentum, because the rotation periods are measured directly and are more accurate than the rotation velocities, which are affected by the projection effect. 
Apart from the rotation periods of the stars from our sample, we used the rotation periods for solar-type dwarfs (with masses of $0.9-1.1 M_{\odot}$ ) in the clusters IC 2602 (an age of $30 \mathrm{Myr}$ ), Alpha Persei (50 Myr), the Pleiades (100 Myr), and the Hyades (700 Myr) from Pizzolato et al. (2003). The rotation period is plotted against the age for the stars from our sample and for dwarfs from the clusters listed above in Fig. 6. To interpret the observational data, we applied a simple model that we had already used previously (see Bouvier et al. 1997) and that is described in detail in Bouvier (1994) and Bouvier and Forestini (1994). This model describes the presumed evolution of the rotation period for a solar-mass star as it evolves from the $\mathrm{T}$ Tauri phase to the Sun's age. We made the following assumptions when calculating this model:

(1) The initial rotation period is 8 days, as is observed in many young T Tauri stars.

(2) Solid-body rotation of the star $(d \Omega / d r=0)$ during its PMS and MS evolution.

(3) The existence of a magnetospheric interaction between the star and the disk: all the time the star is surrounded by the disk, it evolves with a constant angular velocity $(d \Omega / d t=0$, i.e., $\left.V(t)=V_{o} R(t) / R_{o}\right)$.

(4) The evolution of the star's angular momentum under the action of a stellar wind after the loss of its disk: the braking law $d J / d t \propto \Omega^{2}$ for rapidly rotating stars $\left(>8 \mathrm{~km} \mathrm{~s}^{-1}\right)$ and $d J / d t \propto \Omega^{3}$ for slowly rotating stars $\left(\leq 8 \mathrm{~km} \mathrm{~s}^{-1}\right)$.

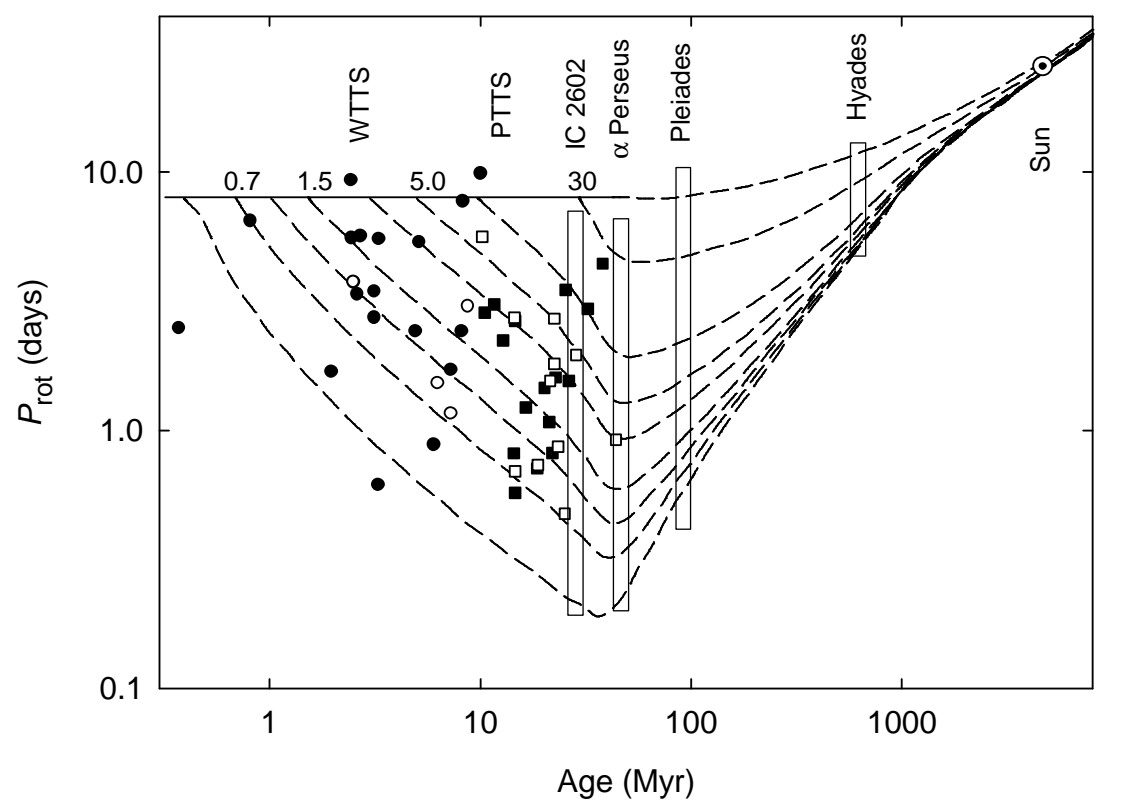

Figure 6: Rotation period versus age. WTTS with a reliable and unreliable evolutionary status are designated by the black and white circles, respectively. PTTS with a reliable and unreliable evolutionary status are marked by the black and white squares, respectively. The light rectangles indicate the positions of cluster dwarfs with various ages. The upper solid horizontal line was calculated for a star rotating with a constant period of 8 days and interacting with the protoplanetary disk during the entire PMS stage (50 Myr). Each dashed line indicates the evolution of the stellar rotation period depending on the specific instant of time when the magnetospheric connection with the disk is lost. These correspond to the following instants of time: $0.4,0.7,1.0,1.5,3.0,5.0$, 10, 30, and $50 \mathrm{Myr}$.

At present, it is commonly assumed that young stars evolve on their convective tracks without spin-up during all the time they remain in interaction with the protoplanetary disks. Each dashed curve shown in Fig. 6 starts at a certain time of the star's PMS evolution: the starting point of the curve corresponds to the age when the magnetospheric connection between the star and its disk ceases. Thereafter, the star begins to rotate freely, remaining under the 
action of only the braking wind, which carries away part of the angular momentum.

The first dashed curve in Fig. 6 starts at an age of 0.4 Myr. At this time, the solar-mass star is slightly below the so-called birth line in the region of the HP diagram where CTTS are actually observed (Kenyon and Hartmann 1995). The starting points of the remaining dashed lines shown in Fig. 6 correspond to ages of 0.7, 1.0, 1.5, 3.0, 5.0, 10, 30, and $50 \mathrm{Myr}$, respectively.

There is a large scatter of rotation periods for all ages for both WTTS and PTTS. According to the proposed model, this scatter can be explained by different active star-disk interaction times. Most of the stars from our sample lie above the track corresponding to an age of 0.7 Myr and below the track corresponding to an age of $10 \mathrm{Myr}$. It is within this time interval that an active star-protoplanetary disk interaction occurs.

Thus, the observed spin-up from the youngest WTTS to PTTS within the time scale from 1 to $40 \mathrm{Myr}$ is well explained by a rapid decrease in the moment of inertia, while the angular momentum losses are moderate. It should be noted that there also exist other models based on completely different ideas that also predict spin-up for stars on PMS radiative tracks (Soderblom et al. 1993; Keppens et al. 1995; Cameron et al. 1995). These models are based on the idea of the so-called saturated dynamo suggesting the absence of an active core-shell interaction instead of uniform internal rotation.

\section{CONCLUSIONS}

We analyzed a sample of 74 magnetically active stars toward the Taurus-Auriga SFR. Based on accurate data on their basic physical parameters obtained from original photometric observations and published data on their proper motions, X-ray luminosities, and equivalent widths of the $H \alpha$ and $\mathrm{Li}$ lines, we refined the evolutionary status and analyzed the relationship between the rotation, mass, and age of these objects. In particular, the following results were obtained.

We showed that 16 well-known PMS objects from our sample are reliable WTTS and 4 PMS objects are reliable PTTS that belong to the Taurus-Auriga SFR. Analysis of the evolutionary status for 50 candidates for PMS stars from Wichmann's list showed that $60 \%$ (30 of 50) of the objects are young stars with ages of 1-40 Myr (14 WTTS and 16 PTTS) that could belong to the Taurus-Auriga SFR with a high probability. We classified 34\% (17 of 50) of the candidates for PMS stars from Wichmann's list as stars with an unreliable evolutionary status that could be assigned to the population of Gould Belt stars. The remaining three stars with ages of about 100 Myr were classified as ZAMS objects.

As a result of this work, we identified a group of 70 young (1-40 Myr), magnetically active stars with reliable data on their rotation periods, luminosities, radii, and masses toward the Taurus-Auriga SFR. The dependence of the rotation period on mass and age was investigated for 50 PMS stars. We showed that the more massive stars of the sample rotate, on average, faster than the less massive ones. The relatively old PMS stars (PTTS, $t>10$ Myr) rotate, on average, faster than the younger ones (WTTS, $\mathrm{t}<10 \mathrm{Myr}$ ). This result is in good agreement with the theoretical models that predict a decrease in the rotation period when moving toward the ZAMS.

We investigated the evolution of angular momentum for PMS stars in Taurus-Auriga. We showed that the distribution of stars on the rotation period-age diagram could be explained by different active star-disk interaction times. Most of the sample stars ceases to actively interact with their disks on a time scale from 0.7 to $10 \mathrm{Myr}$. The observed spin-up from the youngest WTTS to PTTS within a time scale from 1 to $40 \mathrm{Myr}$ is well explained by a rapid decrease in 
the moment of inertia, while the angular momentum losses are moderate.

The possible relationship between various magnetic activity parameters and rotation for 70 PMS stars toward the Taurus-Auriga SFR will be investigated in the next paper.

\section{REFERENCES}

1. G. Basri, E. L. Martin, and C. Bertout, Astron. Astrophys. 252, 625 (1991).

2. C. Bertout and F. Genova, Astron. Astrophys. 460, 499 (2006).

3. J. Bouvier, ASP Conf. Ser. 64, 151 (1994).

4. J. Bouvier and M. Forestini, in Circumstellar Dust Disk and Planetary Formation, Proceedings of the 10th IAP Meeting, Ed. by P. Ferlet (Frontieres, Gif-sur-Yvette, 1994), p. 347.

5. J. Bouvier, R. Wichmann, K. Grankin, et al., Astron. Astrophys. 318, 495 (1997).

6. A. C. Cameron, C. G. Campbell, and H. Quaintrell, Astron. Astrophys. 298, 133 (1995).

7. A. C. Cameron and C. G. Campbel, Astron. Astrophys. 274, 309 (1993).

8. J.M. Carpenter, Astron. J. 120, 3139 (2000).

9. Duy Cuong Nguyen, Ray Jayawardhana, Marten H. van Kerkwijk, et al., Astrophys. J. 695, 1648 (2009).

10. C. Ducourant, R. Teixeira, J. P. Périé, et al., Astron. Astrophys. 438, 769 (2005).

11. E. D. Feigelson, Astrophys. J. 468, 306 (1996).

12. S. Frink, S. Röoser, R. Neuhäuser, et al. Astron. Astrophys. 325, 613 (1997).

13. M. Gomez, B. F. Jones, L. Hartmann, et al., Astron. J. 104, 762 (1992).

14. K. N. Grankin, S. Yu. Melnikov, J. Bouvier, et al., Astron. Astrophys. 461, 183 (2007).

15. K. N. Grankin, J. Bouvier, W. Herbst, et al., Astron. Astrophys. 479, 827 (2008).

16. K. N. Grankin, Astronomy Letters 39, (4), 251 (2013a).

17. M. Güdel, K. R. Briggs, K. Arzner, et al. Astron. Astrophys. 468, 353 (2007).

18. L.W. Hartmann, D. R. Soderblom, and J. R. Stauffer, Astron. J. 93, 907 (1987).

19. G. H. Herbig, in Can Post-T Tauri Stars Be Found?, Ed. by L. V. Mirzoyan (Publ. House Armenian Acad. Sci., Yerevan, 1978), p. 171.

20. G. H. Herbig, F. J. Vrba, and A. E. Rydgren, Astron. J. 91, 575 (1986).

21. G. H. Herbig and K. R. Bell, Third Catalog of Emission Line Stars of the Orion Population, Lick Obser. Bull. (Lick Observ., Santa Cruz, 1988).

22. S. J. Kenyon and L. Hartmann, Astrophys. J. Suppl. Ser. 101, 117 (1995).

23. S. J. Kenyon, M. Gómez, and B. A. Whitney, in Handbook of Star Forming Regions, Vol. 1: The Northern Sky, Ed. by Bo Reipurth (ASP Monograph Publ., 2008), vol. 4, p. 405.

24. R. Keppens, K. B. MacGregor, and P. Charbonneau, Astron. Astrophys. 294, 469 (1995).

25. A. Konigl, Astrophys. J. 370, L39 (1991).

26. A. Magazzù, R. Rebolo, and Y. V. Pavlenko, Astrophys. J. 392, 159 (1992).

27. E. L. Martin, R. Rebolo, A. Magazzù, et al., Astron. Astrophys. 282, 503 (1994).

28. E. L. Martin, Astron. Astrophys. 321, 492 (1997). 
29. E. L. Martin and A. Magazzù, Astron. Astrophys. 342, 173 (1999).

30. S. Matt and R. E. Pudritz, Astrophys. J. 632, L135 (2005).

31. R. Neuhäuser, G. Torres, M. F. Sterzik, et al., Astron. Astrophys. 325, 647 (1997).

32. F. Palla and S. W. Stahler, Astrophys. J. 540, 255 (2000).

33. N. Pizzolato, A. Maggio, G. Micela, et al., Astron. Astrophys. 397, 147 (2003).

34. L. Siess, E. Dufour, and M. Forestini, Astron. Astrophys. 358, 593 (2000).

35. D. R. Soderblom, B. F. Jones, S. Balachandran, et al., Astron. J. 106, 1059 (1993).

36. B. Stelzer and R. Neuhäuser, Astron. Astrophys. 377, 538 (2001).

37. K. M. Strom, F. P. Wilkin, S. E. Strom, et al., Astron. J. 98, 1444 (1989).

38. F. M.Walter, A. Brown, R. D. Mathieu, et al., Astron. J. 96, 297 (1988).

39. R. Wichmann, J. Krautter, J.H.M.M. Schmitt, et al., Astron. Astrophys. 312, 439 (1996).

40. R. Wichmann, G. Torres, C. H. F. Melo, et al., Astron. Astrophys. 359, 181 (2000). 